\title{
Genome-wide association study of low nitrogen tolerance traits at the seedling stage of rapeseed
}

\author{
C.L. ZENG ${ }^{1, *}$, H.P. WAN ${ }^{1}$, X.M. WU ${ }^{2}$, X.G. DAI ${ }^{1}$, J.D. CHEN ${ }^{1}$, Q.Q. JI ${ }^{1}$, and F. QIAN ${ }^{1}$ \\ ${ }^{1}$ Hubei Engineering Research Center for Protection and Utilization of Special Biological Resources in the Hanjiang \\ River Basin, Jianghan University / School of Life Science, Jianghan University, Wuhan 430056, P.R. China \\ ${ }^{2}$ Institute of Oil Crop Research, Chinese Academy of Agricultural Sciences, Wuhan 430078, P.R. China
}

*Corresponding author: E-mail: zengchangli6111@tom.com

\begin{abstract}
The large application of nitrogen fertilizer will cause soil deterioration and pollute the environment. Reduction of nitrogen inputs and maintaining high yields are therefore essential to ensure a more sustainable agriculture. However, little information is available about rapeseed (Brassica napus L.) low nitrogen tolerance. We evaluated low nitrogen tolerance of 304 rapeseed accessions at seedling stage and performed a genome-wide association study to detect low nitrogen tolerance-related quantitative trait loci. A natural population comprising 304 B. napus inbred lines was genotyped with a Brassica $60 \mathrm{~K}$ Illumina Infinium SNP array. Finally, 11 single-nucleotide polymorphisms were associated with 3 low nitrogen tolerance-related traits, which explained $5.79-7.57 \%$ of the phenotypic variation. In addition, three possible candidate genes were located near the genetic region. Our results provide valuable information for understanding the genetic control of rapeseed low nitrogen tolerance at seedling stage and may facilitate a markerbased breeding for rapeseed low nitrogen tolerance.
\end{abstract}

Keywords: Brassica napus, low nitrogen tolerance index, quantitative trait loci, single-nucleotide polymorphisms.

\section{Introduction}

Nitrogen, which is an important component of proteins, nucleic acids, chlorophyll, and some plant hormones, plays an important role in plant metabolism, yield formation, and quality (Wang et al. 2017). Therefore, the profit and loss of nitrogen directly affects the health of plants and ultimately affects the productivity of plants. It is estimated that nearly half of the global grain increase is attributed to the application of nitrogen fertilizers (Tilman et al. 2002). Over the past half century, global agricultural nitrogen use has increased rapidly by 7 times (Hirel et al. 2007, Bouchet et al. 2016a). However, only about half of nitrogen fertilizers can be absorbed by crops, and more than half remains in the soil (Ju et al. 2009, Fan et al. 2012) and causes serious ecological problems such as soil acidification or water eutrophication (Galloway et al. 2008). Therefore, studying the genetic mechanism of crop low nitrogen tolerance traits and using modern biological techniques to breed cultivars with high tolerance to low nitrogen is important for reducing the use of nitrogen fertilizer (Tilman et al. 2002).

Rapeseed (Brassica napus L., genome AACC, 2n = 38 ) is one of the most important oil crops in the world. Rapeseed is a very suitable crop for wheat, rice, and barley rotation, exerting a strong positive influence on yields of subsequent cereals (Christen et al. 1992, Christen and Sieling 1993). However, rapeseed requires more nitrogen fertilizer than other crops (Rathke et al. 2005). Although rapeseed can absorb a large amount of nitrogen from the soil, it has always been considered as a crop with low nitrogen use efficiency and low seed production

$\overline{\text { Received }} 19$ July 2020, last revision 28 September 2020 accepted 5 October 2020.

Abbreviations: GLM - general linear model; GWAS - genome-wide association study; LL - leaf length; LNT - low nitrogen tolerance index; LW - leaf width; MLM - the mixed linear model; PH - plant height; SFM - shoot fresh mass; QTL - quantitative trait locus; SNP - single nucleotide polymorphism.

Acknowledgments: This research was supported by a grant from the National Key Research and Development Plan Project (Grant No. 2016YFD0100202-25) and the Open Projects of Key Laboratory of Biology and Genetic Improvement of Oil Crops (2017012).

Conflict of interest: The authors declare that they have no conflict of interest. 
per unit $\mathrm{N}$ applied, which is around half that for other cereals (Sylvester-Bradley and Kindred 2009). Therefore, understanding the genetic architecture of low nitrogen stress tolerance and improving the nitrogen use efficiency of rapeseed is essential to ensure the competitiveness of the crop at the agronomic, environmental, and economic levels.

Genome-wide association study (GWAS), based on linkage disequilibrium, is a novel method of crop genetic analysis (Nordborg et al. 2002, Zhu et al. 2008). Due to the rapid development of sequencing technology, GWAS has been widely used to dissect the genetic architecture of complex quantitative traits of important cereal crop species, such as rice (Huang et al. 2012, Kumar et al. 2015), wheat (Bellucci et al. 2015), and maize (Tian et al. 2011, Li et al. 2013). In recent years, due to the reduction in the cost of genome sequencing, the cost of SNP marker development has greatly reduced, GWAS has been successfully applied to detect QTLs of complex traits in rapeseed, such as seed mass (Li et al. 2014), harvest index, seed yield per plant (Luo et al. 2015), erucic acid content, glucosinolate content, oil content (Li et al. 2014, Liu et al. 2016), plant height (Li et al. 2016), primary branches (Li et al. 2016, Sun et al. 2016), salt stress (Yong et al. 2015, Wan et al. 2017), cadmium stress (Chen et al. 2018), flowering time (Xu et al. 2015, Wang et al. 2016), and resistance to Sclerotinia stem rot (Wu et al. 2016). For nitrogen use efficiency related traits, GWAS also has successful applications (Wang et al. 2017). However, no QTLs for rapeseed low nitrogen tolerance has been reported.

To better understand the genetic control of low nitrogen tolerance traits in rapeseed, we performed GWAS to identify SNPs associated with low nitrogen tolerancerelated traits with a panel of 304 rapeseed accessions using 19908 SNPs from the Illumina Brassica SNP60 Bead Chip. Seedling growth related traits from the panel were investigated at seedling stage. The objectives of this study were: 1) to obtain a better understanding of the influence of low nitrogen stress on rapeseed seedlings and 2) to perform GWAS to identify the low nitrogen tolerant traitrelated SNPs and genes at the seedling stage of rapeseed development.

\section{Materials and methods}

Plants: A total of 304 rapeseed inbred lines from different geographic origins were selected and successfully phenotyped during seedling growth stages under both normal and low nitrogen conditions. The rapeseed inbred lines were collected from ten countries, 148 rapeseed inbred lines from Asia, 120 from Europe, 19 from North America, 15 from Australia, and 2 from New Zealand (http://11032.cn/W1vbUk).

Low nitrogen tolerance evaluation: In order to minimize the impact of the environment, low nitrogen tolerance traits of rapeseed were evaluated at the seedling stage using a hydroponic system (Fig. 1 Suppl.). We built a hydroponic system $(15 \times 0.6 \times 0.4 \mathrm{~m})$ that consists of more than 3000 rapeseed seedlings. Thirty healthy seeds from all 304 rapeseed lines were germinated according to method descried by Wan et al. (2017). Ten similar seedlings ( 5 plants for normal nitrogen treatment and 5 plants for low nitrogen treatment) from each rapeseed inbred line were selected and individually transferred to the hydroponic system and were grown for another four weeks. During the first week, $0.25 \times$ Hoagland solution was used as the nutrient solution, a $0.5 \times$ Hoagland solution was used in the second week, and finally, a $1 \times$ Hoagland solution was used in the third and fourth weeks. At the beginning of the fifth week, two treatments of normal nitrogen $(6.0 \mathrm{mM})$ and low nitrogen $(0.3 \mathrm{mM})$ were used for the growth of rapeseed, the concentration of each component of nutrient solution is shown in the Table 1 Suppl. The $1 \times$ Hoagland solutions with two nitrogen concentrations were replaced every $7 \mathrm{~d}$. The low nitrogen tolerance evaluation experiment was repeated twice.

After eight weeks, the shoots of the seedlings were harvested for measuring plant height $(\mathrm{PH})$ and shoot fresh mass (SFM), and the fourth functional leaf was selected to measure leaf length (LL) and leaf width (LW). To eliminate the effect of genotype background, low nitrogen tolerance index (LNT) was calculated. The LNT is defined as the ratio of the seedling growth-related traits under low nitrogen condition to the same traits under control condition, as previously described by Lian et al. (2005).

Phenotypic data analysis: Statistical analysis of all phenotypic data was performed with the software SPSS v. 22.0 (IBM, Armonk, NY, USA). Descriptive statistics and Pearson's correlations between the traits were performed using the mean values of all phenotypic data from the traits of 304 rapeseed inbred lines. The figures were made by $R$ software ( $R$ Core Team, Vienna, Austria).

Population structure, principal component analysis, and relative kinship: The genotypes of 304 rapeseed inbred lines were identified using the Brassica $60 \mathrm{~K}$ Illumina ${ }^{\circledR}$ Infinium $S N P$ as described Li et al. (2014), and 19820 high quality SNPs were used to analyze population structure and kinship. The population structure was estimated by admixture software (Alexander et al. 2009). Each $\mathrm{K}$ value, as a putative number of populations set from 1 to 5 . The true $\mathrm{K}$ value was determined by the data crossvalidation error, and the $\mathrm{Q}$ matrix was outputted. Principal component analysis (PCA) was also used to assess the tassel TASSEL 4.0 software (Bradbury et al. 2007). The relative kinship matrix was calculated using $S P A G e D i$ software (Hardy and Vekemans 2002).

Genome-wide association study: Four models were performed for GWAS using TASSEL 4.0 software (Bradbury et al. 2007), the general linear model (GLM) considering $\mathrm{P}(\mathrm{P})$, the general linear model (GLM) considering $\mathrm{Q}$ (Q), the mixed linear model (MLM) considering $\mathrm{P}$ and $\mathrm{K}(\mathrm{P}+\mathrm{K})$, the mixed linear model $(\mathrm{MLM})$ considering $\mathrm{Q}$ and $\mathrm{K}(\mathrm{Q}+\mathrm{K})$. Associations between SNPs and the traits were considered significant at $P<0.0001$ or $-\log _{10} \mathrm{P}>4.0$, 
following published thresholds (Yong et al. 2015, Wan et al. 2017, 2018).

Predicting candidate genes in rapeseed cultivars: $\mathrm{Xu}$ et al. (2016) calculated the linkage disequilibrium decay of rapeseed chromosomes, which was the physical distance on the genome when the value of $r^{2}$ is 0.1 , and the average linkage disequilibrium decay of all the chromosomes is $2.8 \mathrm{Mb}$. Thus, Brassica napus genes, which are orthologous to $A$. thaliana nitrogen efficiency-related genes located within $2.8 \mathrm{Mb}$ of significant SNPs, were regarded as possible candidate genes based on annotation information available in the B. napus reference genome (https://www.genoscope.cns.fr/brassicanapus/data/).

\section{Results}

The extensive phenotypic variation of the 304 rapeseed accessions were observed for PH, LL, LW, and SFM in both low nitrogen stress and control conditions (http://11032.cn/W1vbUk). Mean, minimum, maximum, standard deviation, coefficient of variation of all the phenotypic values were calculated, and presented in Table 1. A significant reduction in PH, LL, LW, and SFM were observed at the low nitrogen stress compared to the control conditions. In the control conditions, PH, LL, LW, and SFM varied $17.00-44.00 \mathrm{~cm}, 18.73-47.00 \mathrm{~cm}$, $5.00-18.53 \mathrm{~cm}$, and $6.71-60.00 \mathrm{~g}$, with averages of $31.75 \mathrm{~cm}, 32.83 \mathrm{~cm}, 9.67 \mathrm{~cm}$, and $27.25 \mathrm{~g}$, respectively. Under low nitrogen stress, the PH, LL, LW, and SFM mean values were $8.60-26.67 \mathrm{~cm}, 7.53-24.67 \mathrm{~cm}$, $3.70-30.73 \mathrm{~cm}$, and $1.33-20.85 \mathrm{~g}$, with averages of $17.42 \mathrm{~cm}, 16.67 \mathrm{~cm}, 6.22 \mathrm{~cm}$, and $7.68 \mathrm{~g}$, respectively. All the traits under low nitrogen and normal nitrogen were consistent with a continuous distribution (Fig. 1).

To reduce the impact of different genotypes, the low nitrogen tolerance indices (LNT) of PH, LL, LW, and SFM were used. The LNT-PH, LNT-LL, LNT-LW, and LNT-SFM varied between $0.32-0.94,0.18-0.91$, $0.34-0.93$, and $0.05-0.89$, with averages of $0.56,0.52$, $0.65,0.30$, respectively (Table 1 ). Four LNT-traits showed the characteristics of quantitative traits (Fig. 2).

To understand the relationships between four low nitrogen indices LNT-PH, LNT-LL, LNT-LW, and LNTSFM, the Pearson correlations between these traits were analyzed using the means of the 304 rapeseed inbred lines. The results showed that there was a significant correlation $(P<0.01)$ between all the indices (Table 2 Suppl.). The correlation coefficients between all the indices were more than 0.4. Among them, the correlation coefficient between LNT-PH and LNT-LL reached 0.631. The results indicated that four low nitrogen tolerance indices could reflect the inhibition of rape growth under low nitrogen environment to some extent.

Further, we determined the population structure and genetic relatedness in rapeseed cultivars. As $\mathrm{K}$ increased from 1 to 5 , cross-validation error reached the minimum value when $\mathrm{K}$ is 4 (Fig. $3 A$ ). Thus, the association panel was divided into 4 sub-groups, designated Q1, Q3, Q3, and Q4 (Fig. 3C). Fifteen lines were assigned to the Q1, mainly composed of winter rapeseed; 32 lines were assigned to the Q2, mainly composed of spring rapeseed; 104 lines were assigned to the Q3, mainly composed of semi-winter rapeseed; 14 lines were assigned to the Q4, mainly composed of rapeseed from Europe and America; and 139 lines were assigned to mix group (Table 1 Suppl.). The analysis of relative kinship showed that $52.1 \%$ of between-line kinship coefficients equaled 0 , and $87.9 \%$ of kinship coefficients ranged from 0 to 0.2 (Fig. $3 B$ ). Thus, most lines in the panel were very weakly related.

For genome-wide association study a total of 11 significant $\left(P<0.0001\right.$ or $\left.-\log _{10} \mathrm{P}>4.0\right)$ association SNPs for three low nitrogen tolerance indices (LNT-PH, LNT-LL, and LNT-SFM) were identified by four models (Table 2, Fig. 2 Suppl.). No significant association SNP for LNT-LW was identified. Of these, 7, 2, and 3 SNPs were identified for LNT-PH, LNT-LL, and LNT-SFM,

Table 1. Phenotypic variation in growth-related traits and low nitrogen tolerance index. PH - plant height, LL - leaf length, LW - leaf width, SFM - shoot fresh mass, CK - control conditions, LOW - low nitrogen treatment, LNT - low nitrogen tolerance index (which is defined as the ratio of the seedling grow-related traits under low nitrogen conditions to the same traits under control conditions), SD standard deviation, $\mathrm{CV}$ - coefficient of variation; ** - significant differences between treatments at $P \leq 0.01$.

\begin{tabular}{|c|c|c|c|c|c|c|c|}
\hline Trait & Treatment & Min & Max & Range & Mean & SD & $\mathrm{CV}[\%]$ \\
\hline \multirow[t]{3}{*}{$\mathrm{PH}[\mathrm{cm}]$} & LOW & 8.60 & 26.67 & 18.07 & $17.44 * *$ & 3.02 & 17.32 \\
\hline & $\mathrm{CK}$ & 17.00 & 44.00 & 27.00 & 31.78 & 5.87 & 18.46 \\
\hline & LNT & 0.32 & 0.94 & 0.62 & 0.56 & 0.13 & 23.61 \\
\hline \multirow[t]{3}{*}{$\mathrm{LL}[\mathrm{cm}]$} & LOW & 7.53 & 24.67 & 17.14 & $16.68 * *$ & 3.10 & 18.56 \\
\hline & $\mathrm{CK}$ & 18.73 & 47.00 & 28.27 & 32.90 & 5.31 & 16.15 \\
\hline & LNT & 0.18 & 0.91 & 0.73 & 0.52 & 0.12 & 22.59 \\
\hline \multirow[t]{3}{*}{ LW [cm] } & LOW & 3.70 & 10.53 & 6.83 & $6.23 * *$ & 0.89 & 14.33 \\
\hline & $\mathrm{CK}$ & 5.47 & 18.53 & 13.06 & 9.70 & 1.57 & 16.20 \\
\hline & LNT & 0.34 & 0.93 & 0.59 & 0.65 & 0.10 & 16.02 \\
\hline \multirow[t]{3}{*}{ SFM [g] } & LOW & 1.33 & 20.85 & 19.52 & $7.74 * *$ & 3.46 & 44.68 \\
\hline & $\mathrm{CK}$ & 6.71 & 60.00 & 53.29 & 27.35 & 9.57 & 34.99 \\
\hline & LNT & 0.05 & 0.89 & 0.84 & 0.30 & 0.15 & 48.69 \\
\hline
\end{tabular}




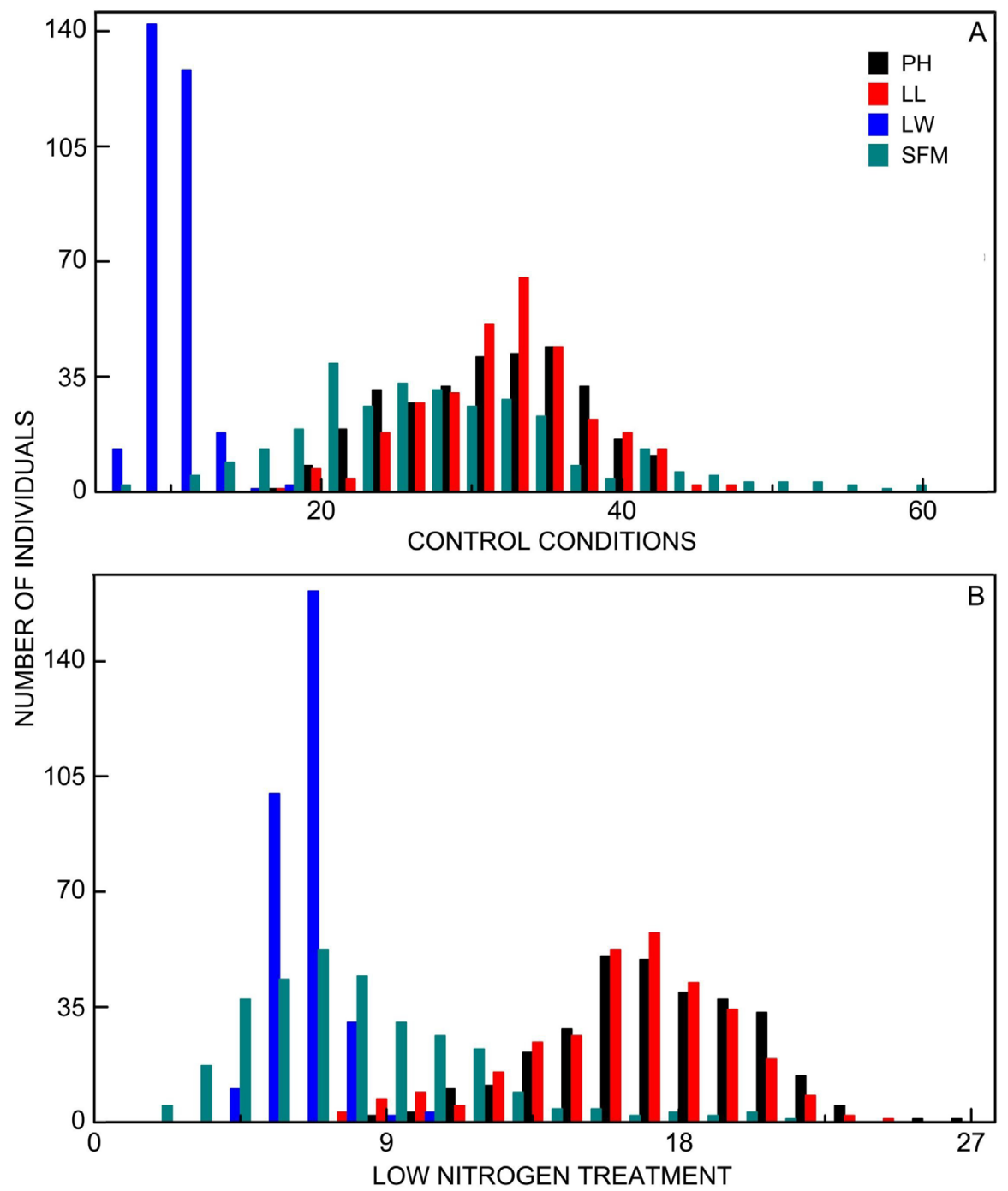

Fig. 1. The diagram of plant height $(\mathrm{PH})[\mathrm{cm}]$, leaf length (LL) $[\mathrm{cm}]$, leaf width (LW) $[\mathrm{cm}]$, and shoot fresh mass (SFM) [g] among the association panel of 304 accessions grown under control conditions $(A)$ and low nitrogen conditions $(B)$.

respectively. One SNP (Bn-A09-p31416695) was simultaneously associated with both LNT-PH and LNTLL.

For LNT-LL, two SNPs were identified on chromosome A09. Bn-A09-p31416695 was identified by P, P+K, Q, and $\mathrm{Q}+\mathrm{K}$ models, and explained $5.82-6.36 \%$ of the phenotypic variation. Bn-A09-p5037318 was identified by $\mathrm{P}, \mathrm{Q}$, and $\mathrm{Q}+\mathrm{K}$ models, and explained $6.60-7.75 \%$ of the phenotypic variation.

For LNT-PH, 7 SNPs were identified on chromosomes A04 and A09. Bn-A04-p5182164 and Bn-A04-p5339433 were identified by $\mathrm{Q}$ models, and explained 6.24 $6.35 \%$ of the phenotypic variation. Bn-A09-p31393136, Bn-A09-p31393314, Bn-A09-p31395071, and BnA09-p31416695 were identified by $\mathrm{P}, \mathrm{Q}$, and $\mathrm{Q}+\mathrm{K}$ models, and explained $5.82-7.15 \%$ of the phenotypic variation.

For LNT-SFM, 3 SNPs were identified on chromosomes A07 and C04. Bn-A07-p1303705, Bn-A07-p9091839 were identified by P and Q models, and explained $5.79-5.82 \%$ of the phenotypic variation. Bn-scaff_15798_1-p42247 was identified by Q models, and explained $6.02 \%$ of the phenotypic variation.

A total of three possible candidate genes were mapped near the SNPs. The BnaA04g07450 was orthologous to $A T 3 G 17820$ (GLN1.3), which encoded a cytosolic glutamine synthetase; BnaA07g09690 was orthologous to AT1G27080 (NRT1.6), which encoded a protein with lowaffinity nitrate transporter activity; BnaA09g47380 was orthologous to AT1G12110 (NRT1.1), which also encoded a protein with low-affinity nitrate transporter activity (Table 3 Suppl.).

\section{Discussion}

The low nitrogen tolerance trait of crops is a quantitative trait regulated by multiple genes, and there are obvious interactions between genes and the environment (Kant et al. 2011, Gao et al. 2015). These factors lead to unstable nitrogen efficiency traits and are susceptible 


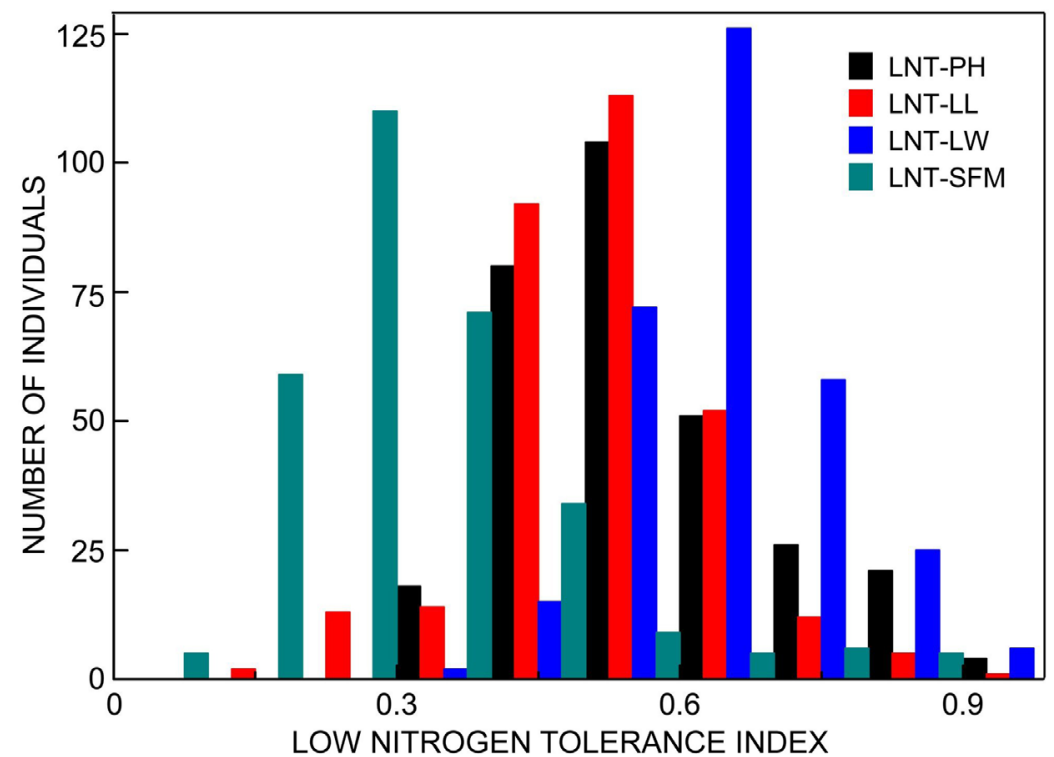

Fig. 2. The distribution of four low nitrogen tolerance indices (LNTs) among the association panel of 304 accessions. The LNT was defined as the ratio of the seedling growth-related traits under low nitrogen conditions to the same traits under control conditions. For explanation of abbreviations see Fig. 1.
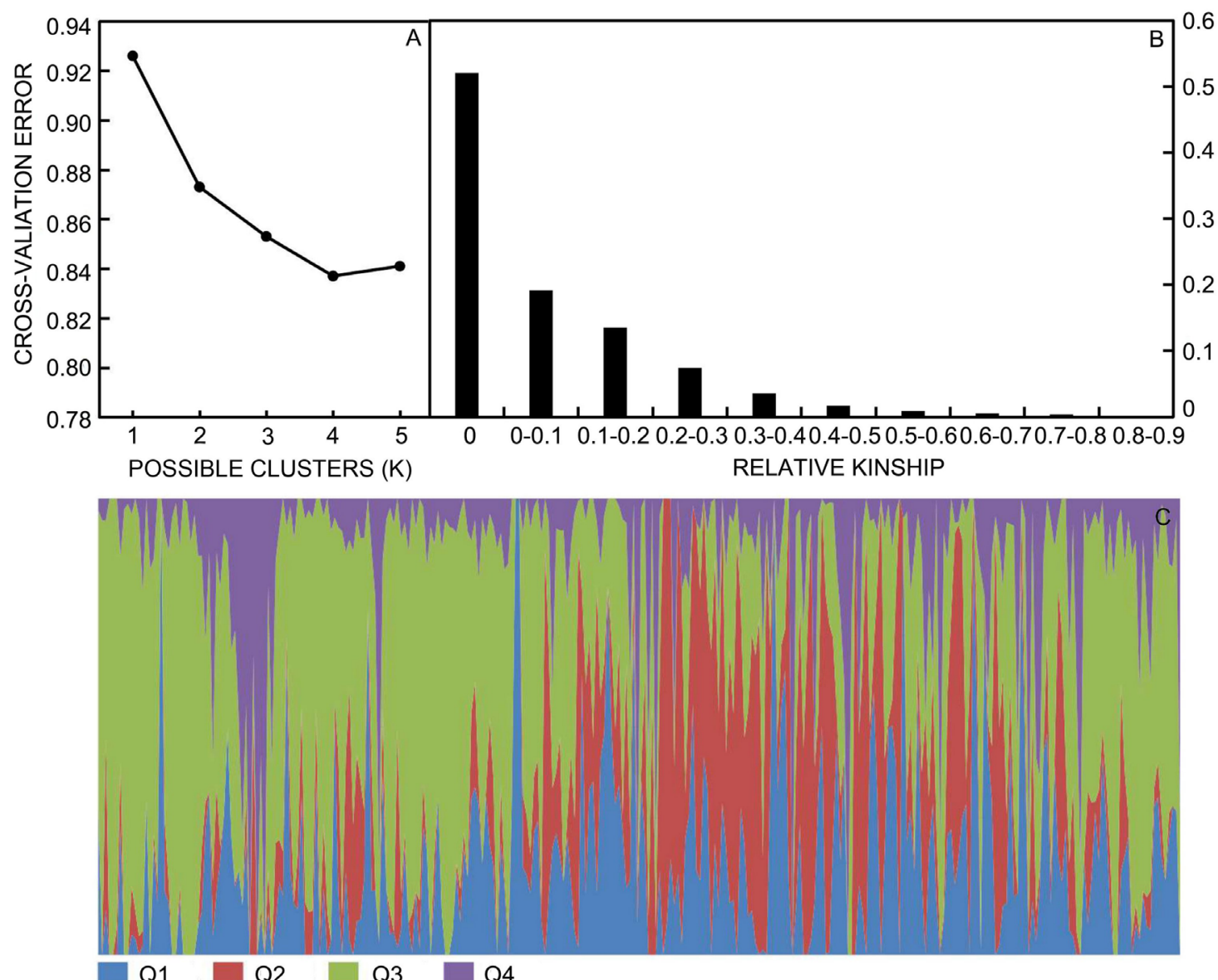

Fig. 3. Analysis of the population structure of 304 rapeseed accessions by Admixtur. A - estimated cross-valiation error of possible clusters (k) from 1 to $5 . B$ - distribution of pairwise relative kinship (304 rapeseed inbred lines). $C$ - population structure based on $\mathrm{K}=$ 4. Each individual is represented by a vertical bar, partitioned into colored segments with the length of each segment representing the proportion of the individual genome. A given group is represented: blue - group 1, red - group 2, green - group 3, and purple - group 4. 
Table 2. The single nucleotide polymorphisms (SNPs) significantly associated with low nitrogen tolerance indices. Allele - base type (A, $\mathrm{T}, \mathrm{C}, \mathrm{G})$ of the SNP site on the allele; $\mathrm{R}^{2}$ - percentage of phenotypic variance explained by SNP; model - general linear model (GLM) considering P and Q, mixed linear model (MLM) considering P and K and Q and K. For other abbreviations see Table 1.

\begin{tabular}{|c|c|c|c|c|c|c|c|}
\hline Trait & Marker name & Chromosome & Site $[\mathrm{b}]$ & Allele & $-\log _{10} P$ & $\mathrm{R}^{2}[\%]$ & Model \\
\hline \multirow[t]{16}{*}{$\overline{\text { LNT-PH }}$} & Bn-A04-p5182164 & A04 & 4051087 & $\mathrm{~A} / \mathrm{G}$ & 4.41 & 6.35 & $\mathrm{Q}$ \\
\hline & Bn-A04-p5339433 & A04 & 3796559 & $\mathrm{~T} / \mathrm{C}$ & 4.33 & 6.24 & Q \\
\hline & \multirow[t]{2}{*}{ Bn-A09-p31393136 } & \multirow[t]{2}{*}{ A09 } & \multirow[t]{2}{*}{29088936} & \multirow[t]{2}{*}{$\mathrm{T} / \mathrm{C}$} & 4.04 & 5.82 & $\mathrm{P}$ \\
\hline & & & & & 4.15 & 5.98 & Q \\
\hline & \multirow[t]{2}{*}{ Bn-A09-p31393314 } & \multirow[t]{2}{*}{ A09 } & \multirow[t]{2}{*}{29089226} & \multirow[t]{2}{*}{$\mathrm{A} / \mathrm{C}$} & 4.33 & 6.22 & $\mathrm{P}$ \\
\hline & & & & & 4.52 & 6.51 & Q \\
\hline & \multirow[t]{2}{*}{ Bn-A09-p31395071 } & \multirow[t]{2}{*}{ A09 } & \multirow[t]{2}{*}{29089789} & \multirow[t]{2}{*}{$\mathrm{A} / \mathrm{C}$} & 4.11 & 6.01 & $\mathrm{P}$ \\
\hline & & & & & 4.28 & 6.26 & Q \\
\hline & \multirow[t]{4}{*}{ Bn-A09-p31416695 } & \multirow[t]{4}{*}{ A09 } & \multirow[t]{4}{*}{29117784} & \multirow[t]{4}{*}{$\mathrm{A} / \mathrm{C}$} & 4.63 & 6.63 & $\mathrm{P}$ \\
\hline & & & & & 4.42 & 6.90 & $\mathrm{P}+\mathrm{K}$ \\
\hline & & & & & 4.96 & 7.12 & Q \\
\hline & & & & & 4.62 & 7.15 & $\mathrm{Q}+\mathrm{K}$ \\
\hline & \multirow[t]{4}{*}{ Bn-A09-p31580606 } & \multirow[t]{4}{*}{ A09 } & \multirow[t]{4}{*}{29271654} & \multirow[t]{4}{*}{$\mathrm{T} / \mathrm{C}$} & 4.34 & 6.26 & $\mathrm{P}$ \\
\hline & & & & & 4.38 & 6.86 & $\mathrm{P}+\mathrm{K}$ \\
\hline & & & & & 4.54 & 6.57 & Q \\
\hline & & & & & 4.59 & 7.13 & $\mathrm{Q}+\mathrm{K}$ \\
\hline \multirow[t]{7}{*}{ LNT-LL } & \multirow[t]{4}{*}{ Bn-A09-p31416695 } & \multirow[t]{4}{*}{ A09 } & \multirow[t]{4}{*}{29117784} & \multirow[t]{4}{*}{$\mathrm{A} / \mathrm{C}$} & 4.06 & 5.82 & $\mathrm{P}$ \\
\hline & & & & & 4.03 & 6.19 & $\mathrm{P}+\mathrm{K}$ \\
\hline & & & & & 4.35 & 6.36 & Q \\
\hline & & & & & 4.09 & 6.35 & $\mathrm{Q}+\mathrm{K}$ \\
\hline & \multirow[t]{3}{*}{ Bn-A09-p5037318 } & A09 & 29633442 & $\mathrm{~T} / \mathrm{C}$ & 4.73 & 6.80 & $\mathrm{P}$ \\
\hline & & A09 & 29633442 & $\mathrm{~T} / \mathrm{C}$ & 5.18 & 7.57 & Q \\
\hline & & A09 & 29633442 & $\mathrm{~T} / \mathrm{C}$ & 4.20 & 6.60 & $\mathrm{Q}+\mathrm{K}$ \\
\hline \multirow[t]{3}{*}{ LNT-SFM } & Bn-A07-p1303705 & A07 & 1237581 & $\mathrm{~T} / \mathrm{G}$ & 4.02 & 5.79 & Q \\
\hline & Bn-A07-p9091839 & A07 & 10553921 & $\mathrm{~T} / \mathrm{C}$ & 4.11 & 5.82 & $\mathrm{P}$ \\
\hline & Bn-scaff_15798_1-p42247 & $\mathrm{C} 04$ & 36662073 & $\mathrm{~A} / \mathrm{G}$ & 4.18 & 6.02 & Q \\
\hline
\end{tabular}

to non-genetic factors (Kant et al. 2011). Therefore, choosing the right evaluation method is the key to obtain accurate phenotypes. Currently, the screening methods for low nitrogen tolerance genotypes of rapeseed use field test methods and potted simulation test methods (Presterl et al. 2003, Barraclough et al. 2010). The pot experiment simulation test can be divided into soil culture test method and hydroponic test method according to the different growth medium. Field trials are generally carried out under natural conditions, which are closer to the actual production but the experimental conditions are complicated (Bouchet et al. 2014). Due to the long time, the growth is affected by various factors, so it is difficult to control the test conditions, and it is not possible to carry out rapid and accurate screening of large quantities. The soil culture pot experiment uses soil as the medium for crop growth, which is closer to actual production. It is the most reliable method for screening low nitrogen tolerant genotypes, but it is difficult to carry out root morphology and physiological and biochemical characteristics. In recent years, hydroponic methods have been successfully applied to the study of nutrient absorption (Huang et al. 2004, Wang et al. 2017). All the nutrients needed for plant growth in hydroponic experiments are supplied manually. The concentration of various nutrient elements in the nutrient solution can be precisely controlled, and the nutrient distribution is relatively uniform (Tocquin et al. 2003). Compared with the lack of air in the solution environment, the concentration is variable, so the hydroponic test has its own limitations. Based on the comparison of the advantages and disadvantages of several screening methods, we selected the hydroponic method to evaluate the low nitrogen tolerance of rapeseed. In order to further increase the homogeneity of the nutrient solution environment of rapeseed seedling growth, we established a culture system that can accommodate more than 3000 rapeseed seedlings, including nutrient solution replenishment system, nutrient solution circulation system, and ventilation system. Through our experiments, we found that this hydroponic system can meet the normal growth of rapeseed and the consistency of the obtained phenotype, and it is ideal for the identification of phenotypes related to nutrient absorption.

The GWAS has become effective tool to identify alleles and loci of crop important traits. However, the results of the correlation analysis are affected by many factors such as species, number of genotypes in population, target traits, marker density, and genetic diversity of population 
(Zhu et al. 2008). Among them, the number of population and its genetic diversity played a major role (Zhao et al. 2007). Thus, collecting germplasm resources with a wide range of genetic sources is an important basis for conducting GWAS. In our study, we collected a natural rapeseed population consisting of 304 rapeseed genotypes originating from 10 countries on 4 continents (http://11032. $\mathrm{cn} / \mathrm{W} 1 \mathrm{vbUk}$ ). Population structure and kinship are main factors of false positive results in GWAS (Flint-Garcia et al. 2005). To reduce the risk of false positives, MLM that considered both $\mathrm{Q}$ and $\mathrm{K}(\mathrm{Q}+\mathrm{K})$, and considered both $\mathrm{P}$ and $\mathrm{K}(\mathrm{P}+\mathrm{K})$, which were more effective than the GLM that considered $\mathrm{Q}$ or $\mathrm{P}$ alone (Stich et al. 2008). However, in some cases, the MLM model is too strict to cause false negative results, and some SNPs locus with low genetic effect could not be detected (Wan et al. 2017). In order to obtain more potential genetic loci, four models ( $\mathrm{P}, \mathrm{Q}, \mathrm{P}+\mathrm{K}$, and $\mathrm{Q}+\mathrm{K}$ ) were selected in this study.

In plant nutrition, it is generally believed that nitrogen efficiency refers to the grain yield produced by plants using effective nitrogen nutrition in the growth medium, which mainly includes two aspects: nitrogen absorption efficiency and nitrogen utilization efficiency (Arrobas et al. 2011). Nitrogen efficiency has different concepts due to the different test subjects and test purposes. However, the nitrogen efficiency has only two points: firstly, low nitrogen tolerance is when the nutrient concentration in the growth medium (soil) is low and the plant has the ability to maintain normal growth and yield; secondly, the high nitrogen sensitivity is an ability to absorb nitrogen fertilizer and obtain higher yield when nitrogen fertilizer is supplied to the growth medium. However, low nitrogen tolerance genotypes are more of a concern for researchers.

Under low nitrogen conditions, genotype differences of crops in nitrogen efficiency are mainly due to nitrogen utilization, while under high nitrogen, absorption efficiency plays a major roles (Moll et al. 1982). Nitrogen use efficiency is more closely related to yield under low nitrogen condition (Lafitte and Edmeades 1994). Thus, plant tolerance to low nitrogen stress is a very complex quantitative trait and regulated by many genes (Lian et al. 2006, Gao et al. 2015). Currently, QTL associated with low nitrogen stress tolerance have been successfully mapped in rice and maize (Liu et al. 2008, Zhang et al. 2015). For example, Zhang et al. (2015) identified a QTL locus related to nitrogen tolerance on chromosome 12 of rice and cloned the TOND1 gene. Cui et al. (2016) identified 109 putative additive QTLs for kernel size and quality characteristics and 49 QTLs for tolerance to low nitrogen stress. At present, there are few genetic studies on the high efficiency of rapeseed nitrogen. For instance, Wang et al. (2017) applied GWAS to study the genetic dissection of root morphological traits related to nitrogen use efficiency in rapeseed, and a total of 23 stable QTLs, which were repeatedly detected in at least two environments or different $\mathrm{N}$ concentrations were identified. However, genetic study on the rapeseed tolerance to low nitrogen has not been reported. In our study, we applied GWAS to identify 11 SNPs related to low nitrogen stress in rapeseed. Comparing our results with QTLs for high efficiency of rapeseed nitrogen (Bouchet et al. 2014, 2016b, Wang et al. 2017), the same genetic locus was not found, suggesting that there were differences in low nitrogen stress and nitrogen efficiency traits. The possible reasons may be as follows: first, the period we identified was the seedling stage, and the period they identified was the whole growth period. Secondly, our study only identified the growth and development traits of the shoots, and they focused on the root traits, indicating that the genetic mechanism of tolerance to low nitrogen environment in the above-ground and under-ground parts of rapeseed may be different. Finally, there may be many ways to cope with low nitrogen environment in rapeseed, such as by increasing nitrogen absorption rate or increasing nitrogen utilization efficiency. Therefore, understanding the relationship between nitrogen efficiency and low nitrogen tolerance in rapeseed will be the focus of our future research.

After plants absorb different forms of nitrogen from the soil by transport proteins, they carry out a series of assimilation processes using different enzymes, and finally complete the nitrogen metabolism. In recent years, researchers have cloned many genes related to plant nitrogen efficiency, including nitrate transport gene (NRT), ammonium ion transport gene (AMT) (Ludewig et al. 2007, Yuan et al. 2007, Hu et al. 2015), that encode enzymes related to nitrogen metabolism in plants (Cai et al.2009) and some other genes (Xu et al. 2012). At present, the molecular basis for the efficient analysis of rapeseed nitrogen have been reported. The expressions of BnNRT1.1, BnNRT2.5, $B n N R T 2.6$, and BnNRT2.7 in the roots of nitrogen-efficient rapeseed are significantly higher than those of nitrogeninefficient germplasms (Wang et al. 2014). In addition, nitrate reductase and glutamine synthetase are important enzymes in the nitrogen assimilation (Sagi et al. 1998). The nitrogen deficiency stress inhibited most genes encoding glutamine synthetase and nitrate reductase. For example, under normal nitrogen conditions, the expressions of $B n G \ln 1,1, B n G \ln 1,2, B n G \ln 1,4$ in the roots of nitrogen efficient germplasm are significantly higher than those of nitrogen inefficient species. As concern the NR family genes, whether under normal nitrogen supply conditions or under nitrogen deficiency conditions, the expressions of $B n N R 1$ in leaves and roots of high-efficiency lines are lower than those in low-efficiency ones. However, under nitrogen-deficient conditions, the expression of $B n N R 2$ in leaves and roots of high-efficiency germplasm was higher than that of low-efficiency germplasm (Wang et al. 2014). What's more, other related genes have also been shown to be related to nitrogen use efficiency. For example, two genes (BnSAG12-1 and BnSAG12-2) coding for SAG12, a cysteine protease implicated in $\mathrm{N}$ remobilization, were observed to reach maximum expression levels at early stages of senescence (Noh and Amasino 1999). In our study, we identified 3 genes (GLN1.3, NRT1.1, and NRT1.6) near the SNPs associated to the low nitrogen stress. Subsequent research needs to continue to verify the function of these genes. 


\section{References}

Alexander, D.H., Novembre, J., Lange, K.: Fast model-based estimation of ancestry in unrelated individuals. - Genome Res. 19: 1655-64, 2009.

Arrobas, M., Parada, M.J., Magalhães, P., Rodrigues M.: Nitrogen-use efficiency and economic efficiency of slowrelease fertilisers applied to irrigated turfs in a mediterranean environment. - Nutr. Cycl. Agroecosyst. 89: 329-339, 2011.

Barraclough, P.B., Howarth, J.R., Jones, J., Lopez, B.R., Parmar, S., Shepherd, C.E., Hawkesford, M.J.: Nitrogen efficiency of wheat: genotypic and environmental variation and prospects for improvement. - Eur. J. Agron. 33: 1-11, 2010.

Bellucci, A., Maria, T.A., Sander, B., Jakob, M., Andersen, S.B., Rasmussen, S.K.: Association mapping in scandinavian winter wheat for yield, plant height, and traits important for second-generation bioethanol production. - Front. Plant Sci. 6: 1046, 2015.

Bouchet, A.S., Nesi, N., Bissuel, C., Bregeon, M., Lariepe, A., Navier, H., Ribiere, N., Orsel, M., Grezes-Besset, B., Renard, M., Laperche, A.: Genetic control of yield and yield components in winter oilseed rape (Brassica napus L.) grown under nitrogen limitation. - Euphytica 199: 183-205, 2014.

Bouchet, A.S., Laperche, A., Bissuel-Belaygue, C., Snowdon, R., Nesi, N., Stahl, A.: Nitrogen use efficiency in rapeseed. A review. - Agron. Sustain. Dev. 36: 38, 2016 a.

Bouchet, A.S., Laperche, A., Bissuel-Belaygue, C., Baron, C., Morice, J.M., Rousseau-Gueutin, M., Dheu, J.E., George, P., Pinochet, X., Foubert, T., Maes, O., Dugué, D., Guinot, F., Nesi, N.: Genetic basis of nitrogen use efficiency and yield stability across environments in winter rapeseed. - BMC Genet. 17: 131, 2016b.

Bradbury, P.J., Zhang, Z., Kroon, D.E., Casstevens, T.M., Ramdoss, Y., Buckler, E.S.: Tassel: software for association mapping of complex traits in diverse samples. - Bioinformatics 23: 2633-2635, 2007.

Cai, H., Zhou, Y., Xiao, J., Li, X., Zhang, Q., Lian, X.: Overexpressed glutamine synthetase gene modifies nitrogen metabolism and abiotic stress responses in rice. - Plant Cell Rep. 28: 527-537, 2009.

Cui, F., Fan, X., Chen, M., Zhang, N., Zhao, C., Zhang, W., Han, J., Ji, J., Zhao, X., Yang, L., Zhao, Z., Tong, Y., Wang, T., Li, J.: QTL detection for wheat kernel size and quality and the responses of these traits to low nitrogen stress. - Theor. appl. Genet. 129: 469-484, 2016.

Chen, L., Wan, H., Qian, J., Guo, J., Sun, C., Wen, J., Yi, B., Ma, C., Tu, J., Song, L., Fu, T., Shen, J.: Genome-wide association study of cadmium accumulation at the seedling stage in rapeseed (Brassica napus L.). - Front. Plant Sci. 9: 375, 2018.

Christen, O., Sieling, K., Hanus, H.: The effect of different preceding crops on the development, growth and yield of winter wheat. - Eur. J. Agron. 1: 21-28, 1992.

Christen, O., Sieling, K.: The effect of different preceding crops on the development, growth and yield of winter barley. - J. Agron. Crop Sci. 171: 114-123, 1993.

Fan, M., Shen, J., Yuan, L., Jiang, R., Chen, X., Davies, W.J., Zhang, F.: Improving crop productivity and resource use efficiency to ensure food security and environmental quality in China. - J. exp. Bot. 63: 13-24, 2012.

Flint-Garcia, S.A., Thuillet, A.C., Yu, J., Pressoir, G., Romero, S.M., Mitchell, S.E., Doebley, J., Kresovich, S., Goodman, M.M., Buckler, E.S.: Maize association population: a highresolution platform for quantitative trait locus dissection. Plant J. 44: 1054-1064, 2005.

Galloway, J.N., Townsend, A.R., Erisman, J.W., Bekunda, M., Cai, Z., Freney, J.R., Martinelli, L.A., Seitzinger, S.P., Sutton,
M.A.: Transformation of the nitrogen cycle: recent trends, questions, and potential solutions. - Science 320: 889-892, 2008.

Gao, K., Chen, F., Yuan, L., Zhang, F., Mi, G.: A comprehensive analysis of root morphological changes and nitrogen allocation in maize in response to low nitrogen stress. - Plant Cell Environ. 38: 740-750, 2015.

Hardy, O., Vekemans, X.: SPAGEDi: a versatile computer program to analyse spatial genetic structure at the individual or population levels. - Mol. Ecol. Notes 2: 618-620, 2002.

Hirel, B., Le Gouis, J., Ney, B., Gallais, A.: The challenge of improving nitrogen use efficiency in crop plants: towards a more central role for genetic variability and quantitative genetics within integrated approaches. - J. exp. Bot. 58: 23692387, 2007.

Hu, B., Wang, W., Ou, S., Tang, J., Li, H., Che, R., Zhang, Z., Chai, X., Wang, H., Wang, Y., Liang, C., Liu, L., Piao, Z., Deng, Q., Deng, K., Xu, C., Liang, Y., Zhang, L., Li, L., Chu, C.: Variation in NRT1.1B contributes to nitrate-use divergence between rice subspecies. - Nat. Genet. 47: 834-838, 2015.

Huang, G., Yi, K.K., Wu, Y.R., Zhu, L., Mao, C.Z., Wu, P.: QTLs for nitrate induced elongation and initiation of lateral roots in rice (Oryza sativa L.). - Plant Soil 263: 229-237, 2004.

Huang, X., Zhao, Y., Wei, X., Li, C., Wang, A., Zhao, Q., Li, W., Guo, Y., Deng, L., Zhu, C., Fan, D., Lu, Y., Weng, Q., Liu, K., Zhou, T., Jing, Y., Si, L., Dong, G., Huang, T., Lu, T., Feng, Q., Qian, Q., Li, J., Han, B.: Genome-wide association study of flowering time and grain yield traits in a worldwide collection of rice germplasm. - Nat. Genet. 44: 32-39, 2012.

Ju, X., Xing, G., Chen, X., Zhang, S., Zhang, L., Liu, X., Cui, Z., Yin, B., Christie, P., Zhu, Z., Zhang, F.: Reducing environmental risk by improving $\mathrm{N}$ management in intensive Chinese agricultural systems. - Proc. nat. Acad. Sci. USA 106: 3041-3046, 2009.

Kant, S., Bi, Y., Rothstein, S.J.: Understanding plant response to nitrogen limitation for the improvement of crop nitrogen use efficiency. - J. exp. Bot. 62: 1499-1509, 2011.

Kumar, V., Singh, A., Amitha Mithra, S.V., Krishnamurthy, S.L., Parida, S.K., Jain, S., Tiwari, K.K., Kumar, P., Rao, A.R., Sharma, S.K., Khurana, J.P., Singh, N.K., Mohapatra, T.: Genome-wide association mapping of salinity tolerance in rice (Oryza sativa). - DNA Res. 22: 133-145, 2015.

Lafitte, H.R., Edmeades, G.O.: Improvement for tolerance to low soil nitrogen in tropical maize I. Selection criteria. - Field Crop. Res. 39: 1-14, 1994.

Li, F., Chen, B., Xu, K., Gao, G., Yan, G., Qiao, J., Li, J., Li, H., Li, L., Xiao, X., Zhang, T., Nishio T., Wu, X.: A genome-wide association study of plant height and primary branch number in rapeseed (Brassica napus). - Plant Sci. 242: 169-177, 2016.

Li, F., Chen, B., Xu, K., Wu, J., Song, W., Bancroft, I., Harper, A.L., Trick, M., Liu, S., Gao, G., Wang, N., Yan, G., Qiao, J., Li, J., Li, H., Xiao, X., Zhang, T., Wu, X.: Genome-wide association study dissects the genetic architecture of seed weight and seed quality in rapeseed (Brassica napus L.). DNA Res. 21: 355-367, 2014.

Li, H., Peng, Z., Yang, X., Wang, W., Fu, J., Wang, J., Han, Y., Chai, Y., Guo, T., Yang, N., Liu, J., Warburton, M.L., Cheng, Y., Hao, X., Zhang, P., Zhao, J., Liu, Y., Wang, G., Li, J., Yan, J.: Genome-wide association study dissects the genetic architecture of oil biosynthesis in maize kernels. - Nat. Genet. 45: 43-50, 2013.

Lian, X., Wang, S., Zhang, J., Feng, Q., Zhang, L., Fan, D., Li, X., Yuan, D., Han, B., Zhang, Q.: Expression profiles of 10,422 genes at early stage of low nitrogen stress in rice assayed using a cDNA microarray. - Plant mol. Biol. 60: 617 631,2006 
Lian, X., Xing, Y., Yan, H., Xu, C., Li, X., Zhang, Q.: QTLs for low nitrogen tolerance at seedling stage identified using a recombinant inbred line population derived from an elite rice hybrid. - Theor. Appl. Genet. 112: 85-96, 2005.

Liu, S., Fan, C., Li, J., Cai, G., Yang, Q., Wu, J., Yi, X., Zhang, C., Zhou, Y.: A genome-wide association study reveals novel elite allelic variations in seed oil content of Brassica napus. Theor. Appl. Genet. 129: 1203-1215, 2016.

Liu, Z.H., Xie, H.L., Tian, G.W., Chen, S.J., Wang, C.L., Hu, Y.M., Tang, J.H.: QTLs mapping of nutrient components in maize kernels under low nitrogen conditions. - Plant Breed. 127: 279-285, 2008.

Ludewig, U., Neuhauser, B., Dynowski, M.: Molecular mechanisms of ammonium transport and accumulation in plants. - FEBS Lett. 581: 2301-2308, 2007.

Luo, X., Ma, C., Yue, Y., Hu, K., Li, Y., Duan, Z., Wu, M., Tu, J., Shen, J., Yi, B., Fu, T.: Unravelling the complex trait of harvest index in rapeseed (Brassica napus L.) with association mapping. - BMC Genomics 16: 379, 2015.

Moll, R.H., Kamprath, E.J., Jackson, W.A.: Analysis and interpretation of factors which contribute to efficiency of nitrogen utilization. - Agron. J. 74: 562-564, 1982.

Noh, Y., Amasino, R.: Regulation of developmental senescence is conserved between Arabidopsis and Brassica napus. - Plant mol. Biol. 41: 195-206, 1999.

Nordborg, M., Tavare, S.: Linkage disequilibrium: what history has to tell us. - Trends Genet. 18: 83-90, 2002.

Presterl, T., Seitz, G., Landbeck, M., Thiemt, E.M., Schmidt, W., Geiger, H.H.: Improving nitrogen-use efficiency in European maize. - Crop Sci. 43: 1259-1265, 2003.

Rathke, G.W., Christen, O., Diepenbrock, W.: Effects of nitrogen source and rate on productivity and quality of winter oilseed rape (Brassica napus L.) grown in different crop rotations. Field Crops Res. 94: 103-113, 2005.

Sagi, M., Dovrat, A., Kipnis, T., Lips, H.: Nitrate reductase, phosphoenolpyruvate carboxylase, and glutamine synthetase in annual ryegrass as affected by salinity and nitrogen. - J. Plant Nutr. 21: 707-723, 1998.

Stich, B., Mohring, J., Piepho, H.P., Heckenberger, M., Buckler, E.S., Melchinger, A.E.: Comparison of mixed-model approaches for association mapping. - Genetics 178: 17451754, 2008.

Sun, C., Wang, B., Wang, X., Hu, K., Li, K., Li, Z., Li, S., Yan, L., Guan, C., Zhang, J., Zhang, Z., Chen, S., Wen, J., Tu, J., Shen, J., Fu, T., Yi, B.: Genome-wide association study dissecting the genetic architecture underlying the branch angle trait in rapeseed (Brassica napus L.). - Sci. Rep. 6: 33673, 2016.

Sylvester-Bradley, R., Kindred, D.R.: Analysing nitrogen responses of cereals to prioritize routes to the improvement of nitrogen use efficiency. - J. exp. Bot. 60: 1939-1951, 2009.

Tian, F., Bradbury, P.J., Brown, P.J., Hung, H., Sun, Q., FlintGarcia, S., Rocheford, T.R., McMullen, M.D., Holland, J.B., Buckler, E.S.: Genome-wide association study of leaf architecture in the maize nested association mapping population. - Nat. Genet. 43, 159-162, 2011.

Tilman, D., Cassman, K.G., Matson, P.A., Naylor, R., Polasky, S.: Agricultural sustainability and intensive production practices.
- Nature 418: 671-677, 2002.

Tocquin, P., Corbesier, L., Havelange, A., Pieltain, A., Kurtem, E., Bernier, G., Périlleux, C.: A novel high efficiency, low maintenance, hydroponic system for synchronous growth and flowering of Arabidopsis thaliana. - BMC Plant Biol. 3: 2, 2003.

Wan, H., Chen, L., Guo, J., Li, Q., Wen, J., Yi, B., Ma, C., Tu, J., $\mathrm{Fu}, \mathrm{T}$., Shen, J.: Genome-wide association study reveals the genetic architecture underlying salt tolerance-related traits in rapeseed (Brassica napus L.). - Front. Plant Sci. 8: 593, 2017.

Wan, H., Wei, Y., Qian, J., Gao, Y., Wen, J., Yi, B., Ma, C., Tu, J., Fu, T., Shen, J.: Association mapping of salt tolerance traits at germination stage of rapeseed (Brassica napus L.). Euphytica 214: 190, 2018.

Wang, J., Dun, X., Shi, J., Wang, X., Liu, G., Wang, H.: Genetic dissection of root morphological traits related to nitrogen use efficiency in Brassica napus L. under two contrasting nitrogen conditions. - Front. Plant Sci. 8: 1709, 2017.

Wang, N., Chen, B., Xu, K., Gao, G., Li, F., Qiao, J., Yan, G., Li, J., Li, H., Wu, X.: Association mapping of flowering time QTLs and insight into their contributions to rapeseed growth habits. - Front. Plant Sci. 7: 338, 2016.

Wang, G., Ding, G., Li, L., Cai, H., Ye, X., Zou, J., Xu, F.: Identification and characterization of improved nitrogen efficiency in interspecific hybridized new-type Brassica napus. - Ann. Bot. 114: 549-559, 2014.

Wu, J., Zhao, Q., Liu, S., Shahid, M., Lan, L., Cai, G., Zhang, C., Fan, C., Wang, Y., Zhou, Y.: Genome-wide association study identifies new loci for resistance to sclerotinia stem rot in Brassica napus. - Front. Plant Sci. 7: 1418, 2016.

Xu, G., Fan, X., Miller, A.J.: Plant nitrogen assimilation and use efficiency. - Annu. Rev. Plant. Biol. 63:153-182, 2012.

Xu, L., Hu, K., Zhang, Z., Guan, C., Chen, S., Hua, W., Li, J., Wen, J., Yi, B., Shen, J., Ma, C., Tu, J., Fu, T.: Genome-wide association study reveals the genetic architecture of flowering time in rapeseed (Brassica napus L.). - DNA Res. 23: 43-52, 2016.

Yong, H.Y., Wang, C., Bancroft, I., Li, F., Wu, X., Kitashiba, H., Nishio, T.: Identification of a gene controlling variation in the salt tolerance of rapeseed (Brassica napus L.). - Planta 242: 313-326, 2015.

Yuan, L., Loque, D., Kojima, S., Rauch, S., Ishiyama, K., Inoue, E., Takahashi, H., von Wiren, N.: The organization of highaffinity ammonium uptake in Arabidopsis roots depends on the spatial arrangement and biochemical properties of AMT1type transporters. - Plant Cell 19: 2636-2652, 2007.

Zhang, Y., Tan, L., Zhu, Z., Yuan, L., Xie, D., Sun, C.: TOND1 confers tolerance to nitrogen deficiency in rice. - Plant J. 81: 367-376, 2015.

Zhao, J., Paulo, M.J., Jamar, D., Lou, P., Van Eeuwijk, F., Bonnema, G., Vreugdenhil, D., Koornneef, M.: Association mapping of leaf traits, flowering time, and phytate content in Brassica rapa. - Genome 50: 963-973, 2007.

Zhu, C.S., Gore, M., Buckler, E.S., Yu, J.M.: Status and prospects of association mapping in plants. - Plant Genome 1: 5-20, 2008. 